\title{
Intratumoral heterogeneity and biomarkers for transformation into small cell lung carcinomas from lung adenocarcinomas
}

\author{
Hyun Lee, Sang-Won Um \\ Department of Medicine, Samsung Medical Center, Division of Pulmonary and Critical Care Medicine, Sungkyunkwan University School of \\ Medicine, Seoul, South Korea \\ Correspondence to: Sang-Won Um, MD, MPH, PhD. Department of Medicine, Samsung Medical Center, Division of Pulmonary and Critical Care \\ Medicine, Sungkyunkwan University School of Medicine, 81 Irwon-ro, Gangnam-gu, Seoul 06351, Korea. Email: sangwonum@skku.edu. \\ Provenance: This is an Invited Editorial commissioned by Section Editor Dr. Tianxiang Chen (Department of Thoracic Oncology, Shanghai Chest \\ Hospital, Shanghai Jiao Tong University, Shanghai, China). \\ Comment on: Lee JK, Lee J, Kim S, et al. Clonal history and genetic predictors of transformation into small-cell carcinomas from lung \\ adenocarcinomas. J Clin Oncol 2017;35:3065-74.
}

Submitted Sep 27, 2017. Accepted for publication Oct 05, 2017.

doi: $10.21037 /$ jtd.2017.10.91

View this article at: http://dx.doi.org/10.21037/jtd.2017.10.91

Recent advances in next-generation sequencing have opened a new prospect in the field of cancer genomics and cancer evolution. Using next-generation sequencing, researchers have revealed that the occurrence of somatic events such as mutations and copy number alterations is associated with tumor evolution and genomic diversity in tumors, which is known as tumor heterogeneity (1). Tumor heterogeneity includes interpatient tumor heterogeneity (2), intratumoral heterogeneity $(3,4)$, intermetastatic heterogeneity $(5-8)$, and intrametastatic heterogeneity (9).

Tumor heterogeneity is a key factor associated with heterogeneous treatment responses including cancer progression, relapse and drug resistance (10). Primary lung cancers harbor a larger mutational burden compared to other cancers, which might be a reason of poor treatment outcomes in patients with primary lung cancer (1,11-13). Intratumoral heterogeneity, characterized by larger fractions of subclonal mutations in primary tumors is related to postsurgical recurrence in localized lung adenocarcinoma (LADC) (3). Another study also showed that intratumoral heterogeneity related to elevated copy number was closely associated with cancer recurrence or death in patients with early stage non-small cell lung cancer (14). Furthermore, the different molecular evolution patterns and tumor heterogeneity in metastatic lymph nodes are associated with the heterogeneous treatment response in patients with advanced primary lung cancer (8).
Tumor heterogeneity also limits the treatment response of targeted therapy in advanced LADC. Under selection pressures by these therapies, subclones with intrinsic or acquired resistance are selected, and these selected tumors drive disease progression (15-17). Another important mechanism in which tumors acquire drug resistance is a phenotypic change. For example, some LADC tumors harboring epidermal growth factor receptor (EGFR) mutations transform into small cell lung cancer (SCLC) following treatment with first- and third-generation EGFR/ tyrosine kinase inhibitors (TKIs) in 5\% of patients (17-20). A recent systemic review showed that the transformation into SCLC from LADC following EGFR/TKI therapy appeared to be a late phenomenon (21). The prognosis after SCLC diagnosis is poor and current treatment strategies derived from primary SCLC seem to be ineffective (21). The inactivation of retinoblastoma protein 1 (RB1) was suggested as a possible mechanism for this phenomenon, but it cannot sufficiently explain the transformation from LADC into SCLC (22).

To answer this question, Lee et al. (23) investigated 21 patients with EGFR mutant-LADC tumors that transformed into SCLC after EGFR/TKIs therapy. They performed whole genome sequencing on nine tumors acquired from four patients at different time points. The sequenced tumors exhibited biallelic inactivation of both RB1 and tumor protein p53 (TP53) from the early LADC 
stages, revealing that the complete inactivation of these two genes is crucial for the transformation from LADC into SCLC. At the molecular level, the inactivation of one parental copy of these genes is caused by diverse mechanisms, including frameshift indels, large deletions, and nonsense or inactivating missense substitutions. The other parental copy is inactivated by whole chromosomalor chromosomal arm-level loss of heterozygosity events. These findings were also validated by immunohistochemical analysis in the stage IV LADC tissues of 75 patients who received EGFR/TKIs. In the validated cohort, an EGFRmutant LADC tumor with complete inactivation of $\mathrm{Rb}$ and p53 was 43 times more likely to be transformed into SCLC from LADC. They further showed that apolipoprotein $\mathrm{B}$ mRNA editing enzyme catalytic polypeptide-like (APOBEC)-induced hypermutation was more frequently found in the mutational spectrum of branches toward SCLC, suggesting that APOBEC-induced hypermutation might play a significant role in the late stage of transformation from LADC into SCLC.

Interestingly, two-dimensional plotting of tumor cell fractions for serially acquired tumors revealed that the clonal divergence of SCLC from LADC cells occurred before the first treatment with EGFR/TKIs (23). There were three definite clusters. The first clusters had both EGFR-activating mutations and inactivating mutations of RB1 and TP53. The second and third clusters had only private mutations for LADCs and transformed SCLCs, respectively. These results indicate that LADC and SCLC clones branch directly out from common ancestors and they coexist before the initiation of EGFR/TKIs. During treatment with first- or third-generation EGRF/TKIs, SCLC clones outgrew LADC clones. Therefore, this study addressed the importance of the intratumoral heterogeneity in the era of targeted therapy. However, the mechanism by which the ancestor cells of transformed SCLC survive for approximately 30 months in the presence of effective EGFR/TKIs is not clear. It is likely that a few cancer cells may remain dormant for a long period of time before overt clinical transformation.

Regarding clinical relevance of this study, the researchers identified potent genomic biomarkers that predict transformation from LADC into SCLC. As the transformation into SCLC is associated with poor treatment outcomes, a preemptive strategy to search for the inactivation of $\mathrm{Rb}$ and $\mathrm{p} 53$ in patients with LADC could help identify and manage LADC patients with a high risk of transformation into SCLC. Additionally, Lee et al. proved that immunohistochemical analysis for $\mathrm{Rb}$ and $\mathrm{p} 53$, a simpler method compared to the whole genome sequencing, could be used as an alternative to identify the inactivation of RB1 and TP53.

\section{Acknowledgements}

None.

\section{Footnote}

Conflicts of Interest: The authors have no conflicts of interest to declare.

\section{References}

1. McGranahan N, Swanton C. Clonal Heterogeneity and Tumor Evolution: Past, Present, and the Future. Cell 2017;168:613-28.

2. Vogelstein B, Papadopoulos N, Velculescu VE, et al. Cancer genome landscapes. Science 2013;339:1546-58.

3. Zhang J, Fujimoto J, Zhang J, et al. Intratumor heterogeneity in localized lung adenocarcinomas delineated by multiregion sequencing. Science 2014;346:256-9.

4. de Bruin EC, McGranahan N, Mitter R, et al. Spatial and temporal diversity in genomic instability processes defines lung cancer evolution. Science 2014;346:251-6.

5. Yachida S, Jones S, Bozic I, et al. Distant metastasis occurs late during the genetic evolution of pancreatic cancer. Nature 2010;467:1114-7.

6. Juric D, Castel P, Griffith M, et al. Convergent loss of PTEN leads to clinical resistance to a PI(3)Kalpha inhibitor. Nature 2015;518:240-4.

7. Engelman JA, Zejnullahu K, Mitsudomi T, et al. MET amplification leads to gefitinib resistance in lung cancer by activating ERBB3 signaling. Science 2007;316:1039-43.

8. Um SW, Joung JG, Lee H, et al. Molecular Evolution Patterns in Metastatic Lymph Nodes Reflect the Differential Treatment Response of Advanced Primary Lung Cancer. Cancer Res 2016;76:6568-76.

9. Gerlinger M, Rowan AJ, Horswell S, et al. Intratumor heterogeneity and branched evolution revealed by multiregion sequencing. N Engl J Med 2012;366:883-92.

10. Greaves $M$. Evolutionary determinants of cancer. Cancer Discov 2015;5:806-20.

11. Roberts SA, Lawrence MS, Klimczak LJ, et al. An APOBEC cytidine deaminase mutagenesis pattern is widespread in human cancers. Nat Genet 2013;45:970-6. 
12. Burns MB, Temiz NA, Harris RS. Evidence for APOBEC3B mutagenesis in multiple human cancers. Nat Genet 2013;45:977-83.

13. Alexandrov LB, Nik-Zainal S, Wedge DC, et al. Signatures of mutational processes in human cancer. Nature 2013;500:415-21.

14. Jamal-Hanjani M, Wilson GA, McGranahan N, et al. Tracking the evolution of non-small-cell lung cancer. $\mathrm{N}$ Engl J Med 2017;376:2109-21.

15. Kosaka T, Yatabe Y, Endoh H, et al. Analysis of epidermal growth factor receptor gene mutation in patients with nonsmall cell lung cancer and acquired resistance to gefitinib. Clin Cancer Res 2006;12:5764-9.

16. Turke AB, Zejnullahu K, Wu YL, et al. Preexistence and clonal selection of MET amplification in EGFR mutant NSCLC. Cancer Cell 2010;17:77-88.

17. Piotrowska Z, Niederst MJ, Karlovich CA, et al. Heterogeneity underlies the emergence of EGFRT790 Wild-Type clones following treatment of T790M-positive cancers with a third-generation EGFR Inhibitor. Cancer Discov 2015;5:713-22.

18. Sequist LV, Waltman BA, Dias-Santagata D, et al. Genotypic and histological evolution of lung cancers

Cite this article as: Lee $\mathrm{H}, \mathrm{Um} \mathrm{SW}$. Intratumoral heterogeneity and biomarkers for transformation into small cell lung carcinomas from lung adenocarcinomas. J Thorac Dis 2017;9(11):4248-4250. doi: 10.21037/jtd.2017.10.91 acquiring resistance to EGFR inhibitors. Sci Transl Med 2011;3:75ra26.

19. Yu HA, Arcila ME, Rekhtman N, et al. Analysis of tumor specimens at the time of acquired resistance to EGFR-TKI therapy in 155 patients with EGFR-mutant lung cancers. Clin Cancer Res 2013;19:2240-7.

20. Oser MG, Niederst MJ, Sequist LV, et al. Transformation from non-small-cell lung cancer to small-cell lung cancer: molecular drivers and cells of origin. Lancet Oncol 2015;16:e165-72.

21. Roca E, Gurizzan C, Amoroso V, et al. Outcome of patients with lung adenocarcinoma with transformation to small-cell lung cancer following tyrosine kinase inhibitors treatment: A systematic review and pooled analysis. Cancer Treat Rev 2017;59:117-22.

22. Niederst MJ, Sequist LV, Poirier JT, et al. RB loss in resistant EGFR mutant lung adenocarcinomas that transform to small-cell lung cancer. Nat Commun 2015;6:6377.

23. Lee JK, Lee J, Kim S, et al. Clonal history and genetic predictors of transformation into small-cell carcinomas from lung adenocarcinomas. J Clin Oncol 2017;35:3065-74. 